\section{Cardiopulmonary Resuscitation Practices in the Netherlands: results from a nationwide survey}

Geertje van Limpt ${ }^{1}$, Marc Schluep ${ }^{1}$, Robert Jan Stolker ${ }^{1}$, Sanne Hoeks ${ }^{1}$ and Henrik Endeman ${ }^{2}$

${ }^{1}$ Department of Anaesthesiology, ${ }^{2}$ Department of Intensive Care Medicine, Erasmus University Medical Centre, Rotterdam, the Netherlands

\section{Background}

The survival rates of in-hospital cardiac arrest (IHCA) are low and vary across countries and hospitals. Previous studies have demonstrated that quality characteristics such as team training and adherence to Advanced Life Support (ALS) guidelines are related to a higher survival probability for patients. Furthermore hospital characteristics like teaching status, size and urban location are associated with differences in mortality after IHCA. The European Resuscitation Council guidelines state that more research is needed in the field of survival and contributing factors.

The Resuscitation Outcome in the Netherlands (ROUTiNE) project is a nationwide initiative aimed at describing outcome of IHCA. Part of this project is a survey on CPR characteristics focussed on use of guidelines, training and organisation in Dutch hospitals.

The goal of this survey is to describe the current resuscitation practices among hospitals in the Netherlands.

\section{Methods}

A web-based survey was distributed to all Dutch hospital organizations $(n=77)$ offering inpatient care. Characteristics were stratified by hospital teaching status.

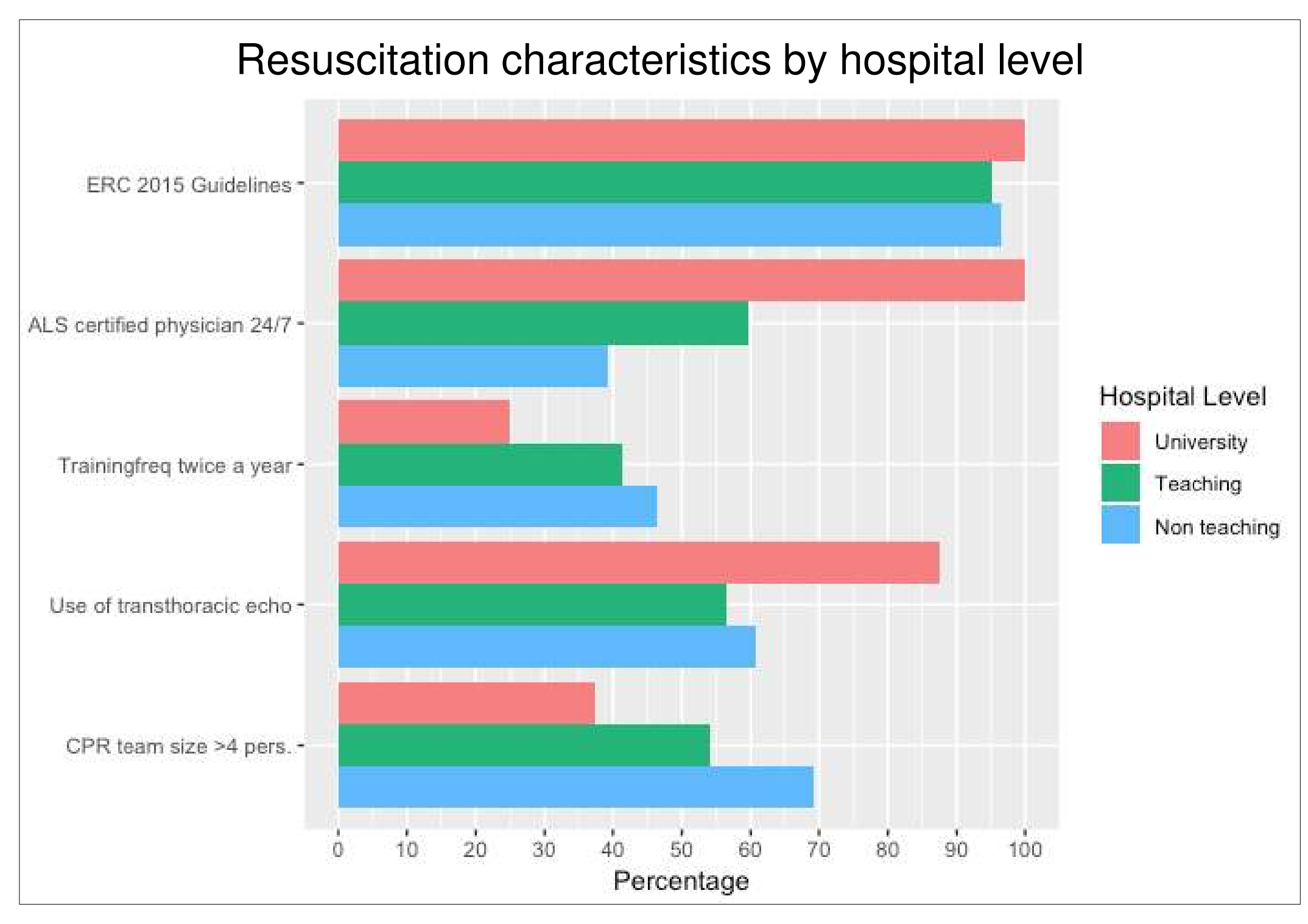

Constitution CPR teams by medical specialty

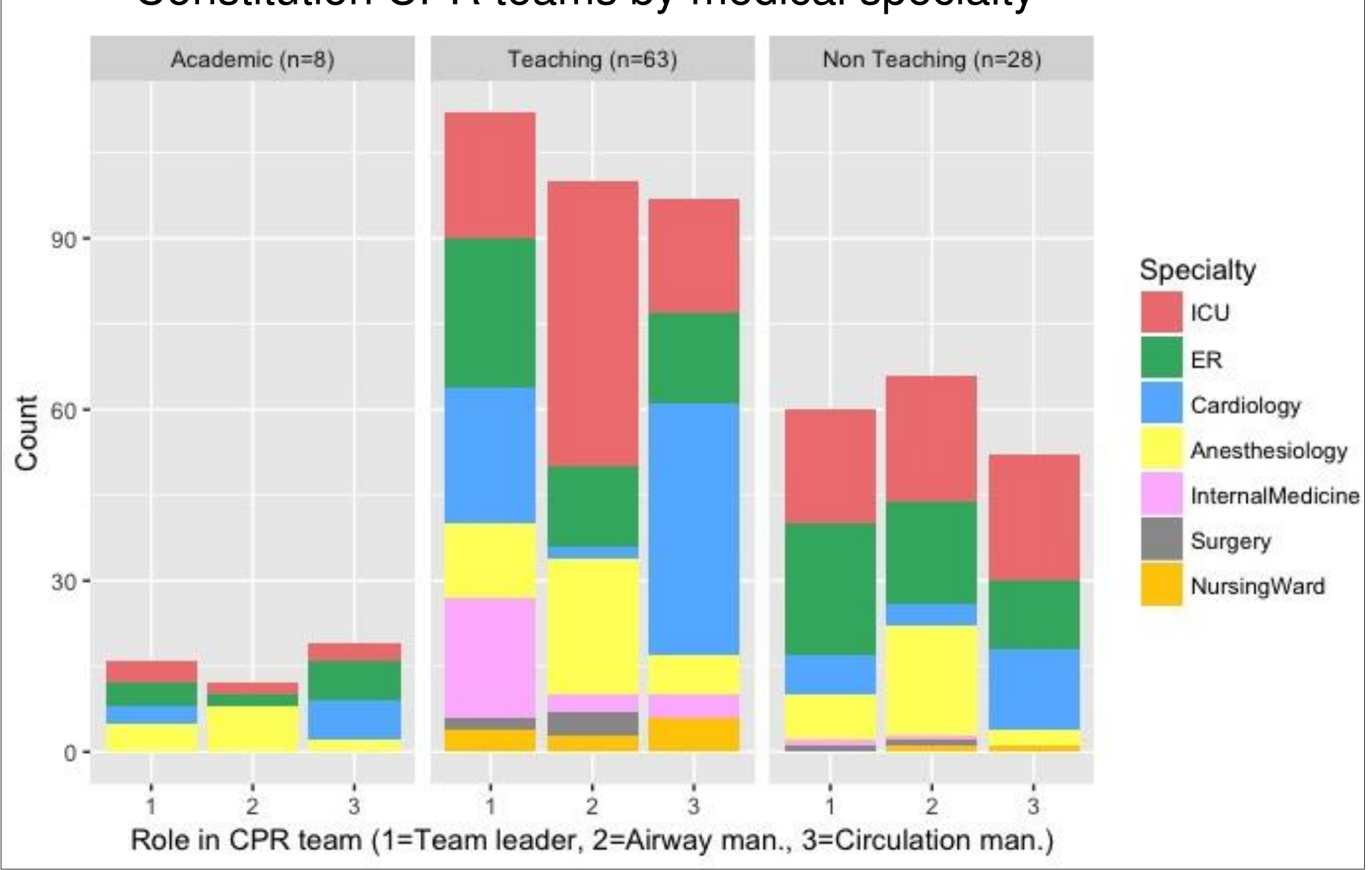

Erasmus MC

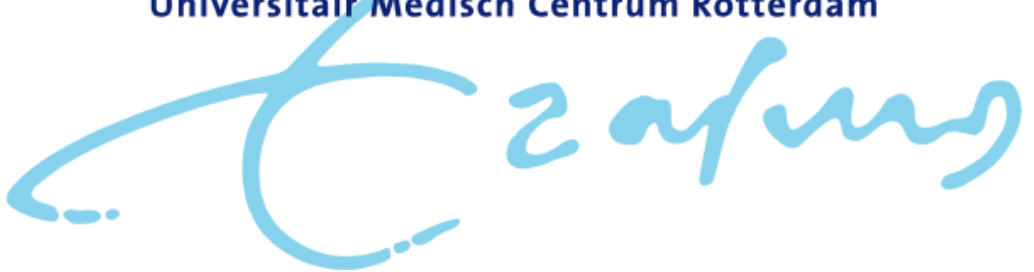

Results

71 out of 77 hospital organizations (92\%) responded the survey, representing 99 locations.

$96 \%$ of the hospitals locations follow the most current European guidelines for advanced life support and $91 \%$ reported the availability of a Rapid Response System. Training frequencies varied with $41 \%$ of the hospital locations training twice a year and 53\% training once a year. The role of CPR team leader and airway manager is most often fulfilled by (resident) anaesthesiologists in academic hospitals (63\%), by Emergency Department professionals in teaching hospitals $(43 \%)$ and by Intensive Care professionals in non-teaching hospitals $(72 \%)$. The role of airway manager is most often attributed to (resident) anaesthesiologists in academic hospitals $(100 \%)$, to Intensive Care professionals in teaching and non-teaching hospitals (82\% and $79 \%$ respectively).

\section{Conclusion}

The majority of Dutch hospitals adhere to the ERC guidelines but there are differences in the presence of an ALS certified physician, intensity of training and the participation of different medical specialties in the fulfilment of roles within the CPR-team. 